\title{
Les Chefs! La SCPH, une aspirante-chef de taille
}

par Myrella Roy

$\mathrm{E}^{\mathrm{n}}$ n 2013, la Société canadienne des pharmaciens d'hôpitaux (SCPH) a touché et retenu le cœur de ses membres en passant par leur estomac, grâce au savoir-faire culinaire unique de la SCPH et des pharmaciens : la combinaison, le mélange et l'altération d'ingrédients professionnels actifs afin de préparer des stratégies de valorisation adaptées, des programmes éducatifs, des occasions de réseautage, des guides de pratiques exemplaires, des subventions de recherche et des hommages à l'excellence. Découvrez les programmes et les services apprêtés par la SCPH pour ses membres et ses parties prenantes à la lecture du menu dégustation ci-après.

Pour son principal défi, la SCPH a dû réaliser un plat de valorisation qui intégrait la préparation des médicaments, le produit vedette de l'année. Elle a ainsi réagi promptement aux répercussions de l'incident, divulgué au début d'avril, au cours duquel des patients de l'Ontario et du Nouveau-Brunswick ont reçu une dose insuffisante d'agents chimiothérapeutiques. Jusqu'au 10 juillet 2013, nous nous sommes affairés dans la cuisine pour partager les énoncés et les lignes directrices pertinentes de la SCPH avec le docteur Jake Thiessen (examinateur indépendant de l'assurance de la qualité dans la chaîne logistique des médicaments anticancéreux en Ontario; consultez son rapport au www.health.gov.on.ca/fr/public/programs/cancer/ drugsupply/docs/report_thiessen_oncology_under-dosing.pdf) et pour commenter les changements de la règlementation et les nouveaux critères d'inspection des installations de préparation de médicaments proposés par l'Ordre des pharmaciens de l'Ontario et le ministère de la Santé et des Soins de Longue Durée de l'Ontario. Nous nous sommes ensuite tirés du défi en équipe comme membre du Sous-groupe de travail du Groupe de travail fédéral-provincial-territorial spécial de Santé Canada sur la surveillance règlementaire du mélange et de la préparation des médicaments : le Sous-groupe de travail a concocté les recommandations relatives aux critères et aux définitions pour délimiter les compétences entre les gouvernements fédéral et provinciaux-territoriaux en matière de fabrication, de préparation en pharmacie et de préparation commerciale des médicaments en cinq semaines au cour de l'été!

Le mets de prédilection de la SCPH, les pénuries de médicaments, a figuré sur le menu l'année durant (www. cshp.ca/advocacy/CSHPspeaks/drugShortages_e.asp). Nous nous sommes associés à l'Association des pharmaciens du
Canada $(\mathrm{APhC})$ et à l'Association médicale canadienne pour mener un sondage auprès de nos membres respectifs à propos de l'incidence des pénuries de médicaments sur les soins des patients et la charge de travail des fournisseurs de soins de santé, et pour diffuser les résultats regroupés aux médias. Comme membre du Comité directeur multilatéral sur les pénuries de médicaments au Canada (coprésidé par Santé Canada et, à titre de meneur du Groupe de travail provincial et territorial sur les pénuries de médicaments, Alberta Health), nous avons contribué à la création et à la parution du Protocole pour la divulgation et la communication des pénuries de médicaments et d'Une boîte à outils pour mieux comprendre les interventions aux pénuries de médicaments au Canada et en accroître la transparence. En juin 2013, nous avons participé au tout premier Sommet international sur les pénuries de médicaments, organisé conjointement par la Fédération internationale pharmaceutique et l'APhC et tenu à Toronto, Ontario. Durant ce forum, 46 représentants de gouvernements, d'organismes professionnels et règlementaires (pharmaciens, médecins et dentistes), de l'industrie et de la chaîne logistique pharmaceutiques, et de groupements de patients ont discuté des causes et des impacts de ce problème mondial complexe ainsi que des solutions pour le résoudre. Six importantes recommandations ont émané du Sommet et devront être considérées par les pays du monde entier, peu importe s'ils ont déjà été affectés par de graves pénuries de médicaments, puisque la structure mondiale de la chaine logistique des médicaments les y expose presque tout autant les uns que les autres.

Lors de l'assemblée générale annuelle en août 2013, les administrateurs de la SCPH ont servi une résolution aux membres de l'association sollicitant l'autorisation de présenter à Industrie Canada une demande de prorogation de la corporation en vertu des dispositions de la nouvelle Loi canadienne sur les organisations à but non lucratif (Loi BNL) et l'approbation des statuts de prorogation et du règlement administratif modifié. En fait, le règlement administratif modifié mijotait depuis cinq ans. Ce travail avait d'abord été motivé par la nécessité de moderniser le règlement administratif afin de mieux définir et encadrer la gouvernance et les pratiques opérationnelles de la SCPH et de ses sections. Entretemps la Loi BNL est entrée en vigueur en octobre 2011. Cette Loi vise à améliorer la transparence et la responsabilité, à doter les organisations de principes de 
gouvernance plus modernes et à conférer certains droits aux membres à l'instar de ceux dont disposent les actionnaires des corporations à but lucratif. Les modifications requises pour respecter la Loi BNL ont donc été incorporées dans l'ébauche du règlement administratif de la SCPH. Selon la résolution adoptée par les membres, le règlement administratif modifié prendra effet le premier mai 2014, bien en avance de la date butoir du 27 octobre 2014 pour se conformer à la Loi BNL.

Les plats principaux de la SCPH ont été accompagnés d'une macédoine d'autres accomplissements collectifs. La portion de ressources liées au projet SCPH 2015 a été généreuse (www.cshp.ca/programs/cshp2015/index_e.asp) : six concours menés par les sections pour promouvoir les réussites; lancement $\mathrm{du}$ forum en ligne, du blogue et du compte Twitter propres au projet SCPH 2015; inauguration du prix pour un projet de résidence en pharmacie d'hôpital afférent aux objectifs SCPH 2015; mesure des progrès publiés dans le Rapport 2011-2012 sur les pharmacies hospitalières canadiennes et affichés sur la page Web du projet SCPH 2015 sous forme de mises à jour nationale et régionales; 13 affiches virtuelles; une troisième trousse à outils (One Dose at a Time: Implementing a Unit-Dose Medication Management System); six webinaires; plusieurs présentations à des congrès; et la troisième édition du concours de vidéos pour les étudiants en pharmacie. Deux publications officielles (Description des activités des pharmaciens dans les dossiers de santé: Lignes directrices et Essais cliniques: Lignes directrices pour les pharmacies des établissements de santé) et deux cours en ligne (Réduction du risque de contamination en pharmacie d'oncologie et Un bilan comparatif des médicaments, voilà la bonne chose à faire) se sont ajoutés à la collection de la SCPH. De nombreuses ressources ont été produites pour aider à sensibiliser à la valeur des pharmaciens hospitaliers et des services pharmaceutiques dans les établissements : une vidéo de deux minutes; un clip médiatique en haute définition de 30 secondes, prêt à l'emploi; ainsi que trois nouvelles affiches et un dépliant. Les caractéristiques démographiques des membres ont été examinées pour la période de 2008-2009 à 2012-2013 et ont été corrélées avec une mesure de la fidélité.

La SCPH a fait un gâteau gargantuesque à l'occasion du $25^{\circ}$ anniversaire de sa Fondation pour la recherche et l'éducation. Les membres ont été invités à participer à la célébration lors de la réception d'ouverture des Séances éducatives d'été 2013 à Calgary en août dernier. La Fondation a subventionné 56 projets de recherche et 7 d'éducation pour une somme totale de 422281 $\$$ depuis 1988. Au cours des dix dernières années, de généreux dons de l'industrie pharmaceutique (totalisant 595922 \$), de la Société et de ses sections (21 391 \$), et des membres à titre individuel (82 757 \$) ont permis à la Fondation de poursuivre ses fins. Vous pouvez aider la Fondation de la SCPH à maintenir cet élan en participant à sa campagne de financement « 25 \$ pour 25 ans " au www.cshpfoundation.ca. Si chaque membre de la SCPH donnait 25 \$, un montant de 65000 \$ serait recueilli pour de futures subventions de recherche et d'éducation. L'une de ces subventions pourrait bien vous être destinée!
La SCPH a aussi été conviée à partager la fortune du pot à la table des consultations menées par diverses parties prenantes : Agrément Canada (sur la pratique organisationnelle requise concernant le bilan comparatif des médicaments aux points de transition des soins telle que proposée dans ses normes modifiées pour les services des urgences), Santé Canada (sur son projet de Règlement sur la maribuana à des fins médicales, sur son Initiative sur l'étiquetage en langage clair pour les médicaments sur et sans ordonnance et les produits biologiques, et sur son Ébauche de ligne directrice révisée - Examen visant à déterminer si le nom médicament est à présentation et à consonance semblables [PSPCS]), l'Institut pour l'utilisation sécuritaire des médicaments au Canada et l'Institut canadien pour la sécurité des patients (sur leur ébauche du Medication Bar Code System Implementation Planning: A Resource Guide), et l'Association nationale des organismes de règlementation de la pharmacie (sur la version définitive de son rapport intitulé Pharmacy Practice Management Systems: Requirements to Support NAPRA Standards of Practice et sur ses versions revues des Professional Competencies for Canadian Pharmacists at Entry to Practice et Professional Competencies for Canadian Pharmacy Technicians at Entry to Practice).

Cette expérience gastronomique n'aurait pas été possible sans les talents culinaires du personnel de la SCPH. Nous avons célébré cinq anniversaires d'embauche marquants : cinq ans pour Olga Chrzanowska (agente du Web), Colleen Drake (agente des publications) et Cathy Lyder (coordonnatrice des affaires professionnelles et du service aux membres), dix ans pour Myrella Roy et vingt ans pour Gloria Day (agente du Conseil canadien de la résidence en pharmacie d'hôpital, et de la valorisation). Nous avons accueilli avec plaisir Robyn Rockwell (agente du service aux membres et des prix) au retour de son congé de maternité et dit adieu à Cheryl Mallory qui avait assumé ce poste à titre intérimaire. Nous avons accordé la permanence à Pamela Saunders (agente de bureau, y compris le soutien administratif au projet SCPH 2015 et au conseil des associés). Nous avons souhaité prompt rétablissement à Laurie Frid (gérante des opérations) et nous avons promu temporairement Desarae Davidson à ce poste de gérante. Nous avons souhaité la bienvenue à Susan Maslin comme agente par intérim des congrès et des réseaux de spécialistes en pharmacie et à Lisa Graziadei comme contrôleure financière temporaire.

Le compte rendu précédent est un festin professionnel susceptible d'impressionner même nos membres les plus épicuriens! Alléchez vos collègues en leur disant combien la $\mathrm{SCPH}$ exerce une influence prépondérante sur les progrès de la pratique en pharmacie dans les établissements de santé et dans d'autres milieux de soins en collaboration au Canada.

Myrella Roy, B. Sc. Phm., Pharm. D., FCCP, est directrice générale de la SCPH. 